\title{
New Refinements and Improvements of Some Trigonometric Inequalities Based on Padé Approximant
}

\author{
Lina Zhang ${ }^{1}$ and Xuesi Ma $\mathbb{D}^{2}$ \\ ${ }^{1}$ School of Computer Science and Technology, Henan Polytechnic University, Jiaozuo, Henan 454000, China \\ ${ }^{2}$ School of Mathematics and Information Science, Henan Polytechnic University, Jiaozuo, Henan 454000, China \\ Correspondence should be addressed to Xuesi Ma; maxuesi@hpu.edu.cn \\ Received 26 March 2020; Accepted 6 May 2020; Published 19 May 2020 \\ Academic Editor: Dimitri Mugnai \\ Copyright (c) 2020 Lina Zhang and Xuesi Ma. This is an open access article distributed under the Creative Commons Attribution \\ License, which permits unrestricted use, distribution, and reproduction in any medium, provided the original work is \\ properly cited. \\ A multiple-point Padé approximant method is presented for approximating and bounding some trigonometric functions in \\ this paper. We give new refinements and improvements of some trigonometric inequalities including Jordan's inequality, \\ Kober's inequality, and Becker-Stark's inequality. The analysis results show that our conclusions are better than the \\ previous conclusions.
}

\section{Introduction}

Inequalities involving trigonometric functions are used in many areas of pure and applied mathematics. Trigonometric inequalities have attracted many researchers. Many improvements of Jordan' inequality [1-11], Kober's inequality
[12-16], and Becker-Stark's inequality $[4,17,18]$ have been obtained.

Recently, Bercu presented a Padé approximant method [19] and obtained the following inequalities:

$$
\begin{gathered}
\frac{-7 x^{2}+60}{3 x^{2}+60}<\frac{\sin (x)}{x}<\frac{11 x^{4}-360 x^{2}+2520}{60 x^{2}+2520}, \quad 0<x<\frac{\pi}{2}, \\
\frac{17 x^{4}-480 x^{2}+1080}{2 x^{4}+60 x^{2}+1080}<\cos (x)<\frac{3 x^{4}-56 x^{2}+120}{4 x^{2}+120}, \quad 0<x<\frac{\pi}{2}, \\
\frac{-28 x^{4}-600 x^{2}+7200}{9 x^{6}+12 x^{4}-3000 x^{2}+7200}<\frac{\tan (x)}{x}<\frac{22 x^{8}-60 x^{6}-4680 x^{4}-237600 x^{2}+2721600}{1020 x^{6}+14040 x^{4}-1144800 x^{2}+2721600}, \quad 0<x<1.5701 .
\end{gathered}
$$


Zhang et al. [20] gave the improvements of inequalities (1)-(3):

$$
\begin{gathered}
\frac{-5 x^{6}+364 x^{4}-9240 x^{2}+60480}{840\left(x^{2}+72\right)} \leq \frac{\sin (x)}{x} \leq \frac{551 x^{4}-22260 x^{2}+166320}{15\left(5 x^{4}+364 x^{2}+11088\right)}, \quad 0 \leq x \leq \frac{\pi}{2}, \\
\frac{-13 x^{6}+660 x^{4}-9720 x^{2}+20160}{360\left(x^{2}+56\right)}<\cos (x)<\frac{313 x^{4}-6900 x^{2}+15120}{13 x^{4}+660 x^{2}+15120}, \quad 0 \leq x \leq \frac{\pi}{2}, \\
\frac{21\left(495-60 x^{2}+x^{4}\right)}{10395-4725 x^{2}+210 x^{4}-x^{6}}<\frac{\tan (x)}{x}<\frac{T_{1}(x)}{105\left(\pi^{2}-4 x^{2}\right) \cdot T_{2}(x)}, \quad 0 \leq x \leq \frac{\pi}{2},
\end{gathered}
$$

where

$$
\begin{aligned}
T_{1}(x)= & \left(\pi^{6}-840 \pi^{4}+75600 \pi^{2}-665280\right) x^{6}+\left(210 \pi^{6}+52920 \pi^{4}-7620480 \pi^{2}+69854400\right) x^{4} \\
& +\left(-17955 \pi^{6}+1323000 \pi^{4}+52390800 \pi^{2}-628689600\right) x^{2}+\left(155925\left(\pi^{4}-112 \pi^{2}+1008\right)\right) \pi^{2} \\
T_{2}(x)= & \left(26 \pi^{4}-2664 \pi^{2}+23670\right) x^{4}+\left(-666 \pi^{4}+73980 \pi^{2}-665280\right) x^{2} \\
& +\left(1485 \pi^{4}-166320 \pi^{2}+1496880\right) .
\end{aligned}
$$

In this paper, we present a multiple-point Padé approximant method for approximating and bounding some trigonometric functions. New refinements and improvements of related trigonometric inequalities are obtained, including Jordan's inequality, Kober's inequality, and Becker-Stark's inequality. We introduce the concept of maximum error to compare our results with the previous methods. The results show that our bounds are tighter than the previous conclusions.

\section{Multiple-Point Padé Approximant Method}

The Padé approximant has been studied in many literature studies [19, 21-24]. In particular, Bercu et al. presented good results of several trigonometric inequalities using the Padé approximant. In this section, we present a multiple-point Padé approximant method. Given a bounded smooth function $f(x)$, let

$$
R(x)=\frac{\sum_{i=0}^{p} a_{i} x^{i}}{1+\sum_{j=1}^{q} b_{j} x^{j}},
$$

be a rational polynomial interpolating of $f(x)$ at multiple points $x_{1}, x_{2}, \ldots, x_{k}$ such that

$$
\begin{aligned}
& E^{\left(i_{1}\right)}\left(x_{1}\right)=0, E^{\left(i_{2}\right)}\left(x_{2}\right)=0, \ldots, E^{\left(i_{k}\right)}\left(x_{k}\right)=0, \\
i_{1}= & 0,1, \ldots, l_{1}, i_{2}=0,1, \ldots, l_{2}, \ldots, i_{k}=0,1, \ldots, l_{k},
\end{aligned}
$$

where $E(x)=\left(1+\sum_{j=1}^{q} b_{j} x^{j}\right) \cdot f(x)-\left(\sum_{i=0}^{p} a_{i} x^{i}\right)$ and $p \geq 0$ and $q \geq 1$ are two given integers. There are $p+q+1$ unknowns in equation (9), $a_{i}$ and $b_{j}, \quad i=0,1,2$, $\ldots, p, j=1,2, \ldots, q$. By selecting suitable values of $l_{1}, l_{2}, \ldots, l_{k}$, we can obtain the polynomial $R(x)$ by solving equation (9).

The general Padé approximant method is a special case of the multiple-point Padé approximant. Here, we just need to consider one point. If $f$ can be written as a formal power series $f(x)=c_{0}+c_{1} x+c_{2} x^{2}+\cdots$, where the coefficients $c_{j}, j=0,1,2, \ldots$, are constant. Taylor's expansion is one of the most common ways to get a power series of a function. The Padé approximant $R_{f}(x)$ of degree $(p, q)$ of the function $f$ is determined by

$$
\left\{\begin{array}{l}
a_{0}=c_{0}, \\
a_{1}=c_{0} b_{1}+c_{1}, \\
a_{2}=c_{0} b_{2}+c_{1} b_{1}+c_{2}, \\
\cdot \\
a_{p}=c_{0} b_{p}+\cdots+c_{p-1} b_{1}+c_{p}, \\
0=c_{p+1}+c_{p} b_{1}+\cdots+c_{p-q+1} b_{q} \\
\cdot \\
\cdot \\
0=c_{p+q}+c_{p+q-1} b_{1}+\cdots+c_{p} b_{q} .
\end{array}\right.
$$

The Padé approximant is considered the "best" approximation of a function by a rational function of a given degree. The rational approximation is also good for series with alternation terms and poor polynomial convergence. 
This is our motivation of using the Padé approximant to approximate trigonometric functions and improve these trigonometric inequalities. Different values of $p$ and $q$ will affect the approximate performance. By selecting suitable values of $p$ and $q$, we can obtain the "best" approximant. Let $(p, q)=(k, k)$, and we can obtain a simple result. The result is a special case of (10).

It is well known that

$$
\tan (x)=\sum_{n=1}^{\infty} T(n) x^{2 n-1}
$$

where

$$
T(n)=\frac{2^{2 n}\left(2^{2 n}-1\right)}{(2 n) !}\left|B_{2 n}\right|
$$

for $x \in(0, \pi / 2), n \in N_{0}$, and $B_{i}$ are Bernoulli's numbers.

Using the Padé approximant and equation (11), we obtain a better approximation of tangent function. Here, we need to pay attention to the value of $c_{j}$ in formula (10). Let $c_{2 j}=0, c_{2 j-1}=T(j)$, and $T(j)$ is given in (12); we can obtain the Padé approximant of $\tan (x)$. In the same way, we can also obtain the Padé approximants of other trigonometric functions.
Table 1 gives the comparison between the Padé approximant and the Taylor series expansion of tangent function. It is easy to see that the maximum approximation error of the Padé approximant is less than the error of the corresponding Taylor polynomial. The advantage of the Padé approximant is more obvious with the increase of the polynomial degree. The bottom row of Table 1 shows the maximum approximation error of the Taylor polynomial is $6.0401 \times 10^{-3}$; however, the maximum approximation error of the Padé approximant is $2.2531 \times 10^{-9}$. At the same time, we can find that the form of the Padé approximant is simpler because of its lower degree.

\section{New Improvements of Jordan's, Kober's, and Becker-Stark's Inequalities}

In this section, we give new improvements of Jordan's, Kober's, and Becker-Stark's inequalities based on the Padé approximant.

Theorem 1. For $\forall x \in \Gamma=[0, \pi / 2]$, we have that

$$
\begin{aligned}
& \frac{3113510400-498960000 x^{2}+22619520 x^{4}-451440 x^{6}+4620 x^{8}-23 x^{10}}{19958400\left(156+x^{2}\right)} \leq \frac{\sin (x)}{x} \\
& \leq \frac{19958400-3144960 x^{2}+136080 x^{4}-2448 x^{6}+19 x^{8}}{181440\left(110+x^{2}\right)} .
\end{aligned}
$$

Proof. Inequality (13) is equivalent to

$$
\left\{\begin{array}{l}
19958400\left(156+x^{2}\right) \sin (x)-\left(3113510400-498960000 x^{2}+22619520 x^{4}-451440 x^{6}+4620 x^{8}-23 x^{10}\right) x \geq 0 \\
181440\left(110+x^{2}\right) \sin (x)-\left(19958400-3144960 x^{2}+136080 x^{4}-2448 x^{6}+19 x^{8}\right) x \leq 0
\end{array}\right.
$$

It is well known that

$$
\begin{aligned}
\alpha(x)-\frac{x^{15}}{1307674368000} & \leq \sin (x) \\
& \leq x-\frac{x^{3}}{6}+\frac{x^{5}}{120}-\frac{x^{7}}{5040}+\frac{x^{9}}{362880}-\frac{x^{11}}{39916800}+\frac{x^{13}}{6227020800}=\alpha(x) .
\end{aligned}
$$

By inequality (15), we have that 


$$
\begin{aligned}
& 19958400\left(156+x^{2}\right) \sin (x)-\left(3113510400-498960000 x^{2}+22619520 x^{4}-451440 x^{6}+4620 x^{8}-23 x^{10}\right) x \\
& \geq 19958400\left(156+x^{2}\right)\left(\alpha(x)-\frac{x^{15}}{1307674368000}\right) \\
& \quad-\left(3113510400-498960000 x^{2}+22619520 x^{4}-451440 x^{6}+4620 x^{8}-23 x^{10}\right) x \\
& =\frac{17 x^{15}}{11351340}\left(54-x^{2}\right) \geq 0, \quad \forall x \in \Gamma, \\
& 181440\left(110+x^{2}\right) \sin (x)-\left(19958400-3144960 x^{2}+136080 x^{4}-2448 x^{6}+19 x^{8}\right) x \\
& \leq 181440\left(110+x^{2}\right) \alpha(x)-\left(19958400-3144960 x^{2}+136080 x^{4}-2448 x^{6}+19 x^{8}\right) x \\
& =\frac{x^{13}}{34320}\left(x^{2}-46\right) \leq 0, \quad \forall x \in \Gamma .
\end{aligned}
$$

Then, the proof of inequality (13) is completed.

Proof. Inequality (17) is equivalent to

Theorem 2. For $\forall x \in \Gamma$, we have that

$$
\begin{aligned}
& \frac{131040-62160 x^{2}+3814 x^{4}-59 x^{6}}{2\left(65520+1680 x^{2}+17 x^{4}\right)} \leq \cos (x) \\
& \leq \frac{1814400-887040 x^{2}+65520 x^{4}-1680 x^{6}+17 x^{8}}{20160\left(90+x^{2}\right)} .
\end{aligned}
$$

$$
\left\{\begin{array}{l}
2\left(65520+1680 x^{2}+17 x^{4}\right) \cos (x)-\left(131040-62160 x^{2}+3814 x^{4}-59 x^{6}\right) \geq 0, \\
20160\left(90+x^{2}\right) \cos (x)-\left(1814400-887040 x^{2}+65520 x^{4}-1680 x^{6}+17 x^{8}\right) \leq 0 .
\end{array}\right.
$$

It is well known that

By inequality (19), we have that

$$
\begin{aligned}
& \beta(x)-\frac{x^{14}}{87178291200} \leq \cos (x) \\
& \leq 1-\frac{x^{2}}{2}+\frac{x^{4}}{24}-\frac{x^{6}}{720}+\frac{x^{8}}{40320}-\frac{x^{10}}{3628800}+\frac{x^{12}}{479001600}=\beta(x) .
\end{aligned}
$$

$$
\begin{aligned}
& 2\left(65520+1680 x^{2}+17 x^{4}\right) \cos (x)-\left(131040-62160 x^{2}+3814 x^{4}-59 x^{6}\right) \\
& \geq 2\left(65520+1680 x^{2}+17 x^{4}\right)\left(\beta(x)-\frac{x^{14}}{87178291200}\right)-\left(131040-62160 x^{2}+3814 x^{4}-59 x^{6}\right) \\
& \quad=\frac{x^{12}}{43589145600}\left(8321040-168168 x^{2}+1414 x^{4}-17 x^{6}\right) \\
& \geq \frac{x^{12}}{43589145600}\left(8321040-168168 \times 2^{2}-17 \times 2^{6}\right) \geq 0, \quad \forall x \in \Gamma, \\
& 20160\left(90+x^{2}\right) \cos (x)-\left(1814400-887040 x^{2}+65520 x^{4}-1680 x^{6}+17 x^{8}\right) \\
& \quad \leq 20160\left(90+x^{2}\right) \beta(x)-\left(1814400-887040 x^{2}+65520 x^{4}-1680 x^{6}+17 x^{8}\right) \\
& \quad=\frac{x^{12}}{23760}\left(-42+x^{2}\right) \leq 0, \quad \forall x \in \Gamma .
\end{aligned}
$$


TABle 1: Comparison between the Padé approximant and the Taylor polynomial for $\tan (x)$.

\begin{tabular}{|c|c|c|c|c|}
\hline \multirow[t]{2}{*}{ Degree } & \multicolumn{2}{|c|}{ Polynomial } & \multicolumn{2}{|c|}{$\begin{array}{c}\text { Maximum approximation } \\
\text { error }\end{array}$} \\
\hline & Padé approximant & Taylor polynomial & Padé & Taylor \\
\hline$[1,1] / 2$ & $x$ & $x$ & $5.5741 \times 10^{-1}$ & $5.5741 \times 10^{-1}$ \\
\hline$[2,2] / 4$ & $\left(3 x /\left(3-x^{2}\right)\right)$ & $x+(1 / 3) x^{3}$ & $5.7408 \times 10^{-2}$ & $2.2407 \times 10^{-1}$ \\
\hline$[3,3] / 6$ & $\left(\left(15 x-x^{3}\right) /\left(15-6 x^{2}\right)\right)$ & $x+(1 / 3) x^{3}+(2 / 15) x^{5}$ & $1.8522 \times 10^{-3}$ & $9.0741 \times 10^{-2}$ \\
\hline$[4,4] / 8$ & $\left(\left(105 x-10 x^{3}\right) /\left(105-45 x^{2}+x^{4}\right)\right)$ & $x+(1 / 3) x^{3}+(2 / 15) x^{5}+(17 / 315) x^{7}$ & $3.0675 \times 10^{-5}$ & $3.6773 \times 10^{-2}$ \\
\hline$[5,5] / 10$ & $\left(\left(945 x-105 x^{3}+x^{5}\right) /\left(945-420 x^{2}+15 x^{4}\right)\right)$ & $\begin{aligned} x+(1 / 3) x^{3} & +(2 / 15) x^{5}+(17 / 315) x^{7} \\
& +(62 / 2835) x^{9}\end{aligned}$ & $3.1725 \times 10^{-7}$ & $1.4903 \times 10^{-2}$ \\
\hline$[6,6] / 12$ & $\begin{array}{c}\left(\left(10395 x-1260 x^{3}+21 x^{5}\right) /\left(10395-4725 x^{2}\right.\right. \\
\left.\left.+210 x^{4}-x^{6}\right)\right)\end{array}$ & $\begin{array}{c}x+(1 / 3) x^{3}+(2 / 15) x^{5}+(17 / 315) x^{7}+ \\
\quad(62 / 2835) x^{9}+(1382 / 155925) x^{11}\end{array}$ & $2.2531 \times 10^{-9}$ & $6.0401 \times 10^{-3}$ \\
\hline
\end{tabular}

Then, the proof of inequality (17) is completed.

where $T_{1}(x)$ and $T_{2}(x)$ are defined in inequality (6).

Theorem 3. For $\forall x \in \Gamma$, we have that

$$
\frac{T_{3}(x)}{T_{4}(x)}<\frac{\tan (x)}{x}<\frac{T_{1}(x)}{105\left(\pi^{2}-4 x^{2}\right) \cdot T_{2}(x)}, \quad 0 \leq x \leq \frac{\pi}{2},
$$

$$
\begin{aligned}
& T_{3}(x)=[(-1679616 \sqrt{3}-2654208 \sqrt{2}-710208) \pi+16376256 \sqrt{3}+21233664 \sqrt{2}-35230464] x^{8} \\
& +\pi[(3079296 \sqrt{3}+4644864 \sqrt{2}+1270080) \pi-27713664 \sqrt{3}-39813120 \sqrt{2}+62923392] x^{7} \\
& +\pi^{2}[(-2297808 \sqrt{3}-3280896 \sqrt{2}-934596) \pi+18869436 \sqrt{3}+30228480 \sqrt{2}-45416304] x^{6} \\
& +\pi^{3}[(894240 \sqrt{3}+1198080 \sqrt{2}+366210) \pi-6632442 \sqrt{3}-11870208 \sqrt{2}+16935408] x^{5} \\
& +\pi^{4}[(-191484 \sqrt{3}-238592 \sqrt{2}-81833) \pi+1272105 \sqrt{3}+2535424 \sqrt{2}-3429916] x^{4} \\
& +\pi^{5}\left[\left(21384 \sqrt{3}+24576 \sqrt{2}+\frac{20295}{2}\right) \pi-252963 \frac{\sqrt{3}}{2}-278528 \sqrt{2}+355544\right] x^{3} \\
& +\pi^{6}\left[\left(-972 \sqrt{3}-1024 \sqrt{2}-\frac{1153}{2}\right) \pi+5103 \sqrt{3}+12288 \sqrt{2}-14572\right] x^{2}+\pi^{8}, \\
& T_{4}(x)=\pi[(-279936 \sqrt{3}-663552 \sqrt{2}-150336) \pi+5038848 \sqrt{3}+2654208 \sqrt{2}-7537536] x^{7} \\
& +\pi^{2}[(606528 \sqrt{3}+1492992 \sqrt{2}+347328) \pi-11617344 \sqrt{3}-5308416 \sqrt{2}+16604352] x^{6} \\
& +\pi^{3}[(-546264 \sqrt{3}-1400832 \sqrt{2}-337140) \pi+11209104 \sqrt{3}+4276224 \sqrt{2}-15206904] x^{5} \\
& +\pi^{4}[(265032 \sqrt{3}+709632 \sqrt{2}+178146) \pi-5860188 \sqrt{3}-1769472 \sqrt{2}+7497936] x^{4} \\
& +\pi^{5}[(-74844 \sqrt{3}-209408 \sqrt{2}-55319) \pi+1790424 \sqrt{3}+395264 \sqrt{2}-2148668] x^{3} \\
& +\pi^{6}\left[\left(12312 \sqrt{3}+35968 \sqrt{2}+\frac{20179}{2}\right) \pi-319059 \sqrt{3}-45056 \sqrt{2}+357852\right] x^{2} \\
& +\pi^{7}\left[\left(-2187 \frac{\sqrt{3}}{2}-3328 \sqrt{2}-1000\right) \pi+30618 \sqrt{3}+2048 \sqrt{2}-\frac{64111}{2}\right] x^{1} \\
& +\pi^{8}\left[\left(81 \frac{\sqrt{3}}{2}+128 \sqrt{2}+\frac{83}{2}\right) \pi-1215 \sqrt{3}+1185\right]+\pi^{9} .
\end{aligned}
$$

Proof. The left side $\left(T_{3}(x) / T_{4}(x)\right)<(\tan (x) / x)$ of inequality (22) is equivalent to

$$
\left\{\begin{array}{l}
e_{3}(x)=\operatorname{sinc}(x)-T_{3}(x) \geq 0, \\
e_{4}(x)=\cos (x)-T_{4}(x) \leq 0
\end{array}\right.
$$

where $\operatorname{sinc}(x)=(\sin (x) / x)$.

It is obvious that 
TABle 2: Comparison of the maximum errors between $\operatorname{sinc}(x)$ and its bounds for different methods.

\begin{tabular}{lrr}
\hline Method & \multicolumn{1}{c}{ Error } & MaxError $_{u}$ \\
\hline Bercu [19] (inequality (1)) & $2.5981 \times 10^{-3}$ & $6.2382 \times 10^{-5}$ \\
Zhang et al. [20] (inequality (4)) & $1.0615 \times 10^{-6}$ & $1.7998 \times 10^{-6}$ \\
Results of this paper (inequality (13)) & $1.3042 \times 10^{-10}$ & $1.3411 \times 10^{-8}$ \\
\hline
\end{tabular}

TABLE 3: Comparison of the maximum errors between $\cos (x)$ and its bounds for different methods.

\begin{tabular}{lrr}
\hline Method & \multicolumn{2}{c}{ Error } \\
& MaxError $_{l}$ & MaxError $_{u}$ \\
\hline Bercu [19] (inequality (2)) & $6.8710 \times 10^{-4}$ & $6.8832 \times 10^{-4}$ \\
Zhang et al. [20] (inequality (5)) & $1.3987 \times 10^{-5}$ & $2.9435 \times 10^{-5}$ \\
Results of this paper (inequality (17)) & $2.9194 \times 10^{-7}$ & $2.0648 \times 10^{-7}$ \\
\hline
\end{tabular}

TABle 4: Comparison of the maximum errors between $\tan (x)$ and its bounds for different methods.

\begin{tabular}{lcr}
\hline Method & \multicolumn{1}{c}{ Error } & MaxError $_{u}$ \\
\hline Zhang et al. [20] (inequality (6)) & MaxError $_{l}$ & $2.7941 \times 10^{-6}$ \\
Results of this paper (inequality (22)) & $2.0690 \times 10^{-1}$ & $2.7941 \times 10^{-6}$ \\
\hline
\end{tabular}

$$
\begin{aligned}
& \operatorname{sinc}^{(9)}(x)=\frac{g(x)}{x^{10}}, \\
& \cos ^{(9)}(x)=-\sin (x) \leq 0,
\end{aligned}
$$

where $g(x)=\left(x^{9}-72 x^{7}+3024 x^{5}-60480 x^{3}+362880 x\right)$ $\cos (x)+\left(-9 x^{8}+504 x^{6}-1520 x^{4}+181440 x^{2}-362880\right)$

$\sin (x)$, and we have $g^{\prime}(x)=-x^{9} \sin (x) \leq 0$; then, $g(x)$ is a monotony decrease function in $(0, \pi / 2), g(x) \geq g(0)=0$. Therefore, $\operatorname{sinc}^{(9)}(x) \leq 0$ for $x \in(0, \pi / 2)$.

By the definition of $e_{3}(x)$ and $e_{4}(x)$, we have

$$
\begin{aligned}
e_{3}(0) & =e_{3}\left(\frac{\pi}{6}\right)=e_{3}\left(\frac{\pi}{4}\right)=e_{3}\left(\frac{\pi}{2}\right)=e_{3}^{\prime}(0)=e_{3}^{\prime}\left(\frac{\pi}{6}\right)=e_{3}^{\prime}\left(\frac{\pi}{4}\right) \\
& =e_{3}^{\prime}\left(\frac{\pi}{3}\right)=0
\end{aligned}
$$

$$
\begin{aligned}
e_{4}(0) & =e_{4}\left(\frac{\pi}{6}\right)=e_{4}\left(\frac{\pi}{4}\right)=e_{4}\left(\frac{\pi}{2}\right)=e_{4}^{\prime}\left(\frac{\pi}{6}\right) \\
& =e_{4}^{\prime}\left(\frac{\pi}{4}\right)=e_{4}^{\prime}\left(\frac{\pi}{3}\right)=e_{4}^{\prime}\left(\frac{\pi}{2}\right)=0,
\end{aligned}
$$

$\forall x \in \Gamma$, and there exist $\xi \in(0, \pi / 2)$ and $\eta \in(0, \pi / 2)$ such that

$$
\begin{aligned}
& e_{3}(x)=\frac{\operatorname{sinc}^{(9)}(\xi)}{9 !} x^{2}\left(x-\frac{\pi}{6}\right)^{2}\left(x-\frac{\pi}{4}\right)^{2}\left(x-\frac{\pi}{3}\right)^{2}\left(x-\frac{\pi}{2}\right) \geq 0, \\
& e_{4}(x)=\frac{\cos ^{(9)}(\eta)}{9 !} x^{2}\left(x-\frac{\pi}{6}\right)^{2}\left(x-\frac{\pi}{4}\right)^{2}\left(x-\frac{\pi}{3}\right)^{2}\left(x-\frac{\pi}{2}\right)^{2} \leq 0,
\end{aligned}
$$

which mean inequality (25) is valid [25]. Then, we have $\left(T_{3}(x) / T_{4}(x)\right)<(\tan (x) / x)$. The proof of $(\tan (x) / x)<$ $\left(T_{1}(x) / 105\left(\pi^{2}-4 x^{2}\right) \cdot T_{2}(x)\right)$ is obtained from [20].

The proof of Theorem 3 is completed.

\section{Conclusions and Analysis}

In this paper, a multiple-point Padé approximant method is presented for approximating and bounding some trigonometric functions. We find that the Padé approximant is a better approximation of trigonometric functions. The conclusion is verified in Table 1 . We give new refinements and improvements of Jordan's, Kober's, and Becker-Stark's inequalities based on the Padé approximant. In order to compare our results with the previous methods, we introduce the concept of the maximum error. The maximum error is the most important index to measure the upper and lower bounds of an inequality. MaxError ${ }_{l}$ denotes the maximum error between a function and its lower bound. MaxError ${ }_{u}$ denotes the maximum error between a function and its upper bound.

Table 2 gives the comparison of the maximum errors between $\operatorname{sinc}(x)$ and its bounds for different methods. It is obvious that the results of this paper are superior to the previous conclusions. The upper and lower bounds of inequality (13) are tighter than inequalities (1) and (4). The results of $\cos (x)$ are presented in Table 3. MaxError Mnd $_{l}$ and MaxError ${ }_{u}$ of inequality (17) is the smallest of three methods in Table 3.

Table 4 gives the comparison of the maximum errors between $\tan (x)$ and its bounds for this paper and Zhang et al.'s paper [20]. Because inequality (3) holds in $(0,1.5701)$, not in $[0, \pi / 2]$, we no longer consider the 
results of literature [19] in the comparison of $\tan (x)$. Table 4 shows that the upper bound of inequality (6) has a maximum error of $2.7941 \times 10^{-6}$; however, the maximum error of the lower bound of inequality (6) is $2.0690 \times 10^{-1}$. This paper greatly improves the lower bound of $\tan (x)$. The maximum error of the lower bound of inequality (22) in this paper reaches $3.0498 \times 10^{-5}$. The maximum error of the lower bound of inequality (22) is far less than that of inequality (6).

\section{Data Availability}

No data were used to support this study.

\section{Conflicts of Interest}

The authors declare that they have no conflicts of interest.

\section{Acknowledgments}

The work was partially supported by the National Natural Science Foundation of China (nos. 11701152 and 11161038), Key Research Project of Colleges and Universities in Henan Province (no. 20A520016), and Ph.D. Foundation of Henan Polytechnic University (no B2017-44).

\section{References}

[1] R. P. Agarwal, Y.-H. Kim, and S. K. Sen, "A new refined Jordan's inequality and its application," Mathematical Inequalities \& Applications, vol. 12, no. 2, pp. 255-264, 2009.

[2] H. Alzer and M. K. Kwong, "Sharp upper and lower bounds for a sine polynomial," Applied Mathematics and Computation, vol. 275, no. 15, pp. 81-85, 2016.

[3] C.-P. Chen and L. Debnath, "Sharpness and generalization of Jordan's inequality and its application," Applied Mathematics Letters, vol. 25, no. 3, pp. 594-599, 2012.

[4] L. Debnath, C. Mortici, and L. Zhu, "Refinements of Jordansteckin and becker-Stark inequalities," Results in Mathematics, vol. 67, no. 1-2, pp. 207-215, 2015.

[5] W. D. Jiang and H. Yun, "Sharpening of Jordan's inequality and its applications," Journal of Inequalities in Pure \& Applied Mathematics, vol. 7, no. 3, 2006.

[6] M. K. Kuo, "Refinements of Jordan's inequality," Journal of Inequalities \& Applications, vol. 2011, no. 1, pp. 1-6, 2011.

[7] S. Wu and L. Debnath, "A new generalized and sharp version of Jordan's inequality and its applications to the improvement of the Yang Le inequality," Applied Mathematics Letters, vol. 19, no. 12, pp. 1378-1384, 2006.

[8] L. Zhu, "Sharpening of Jordan's inequalities and its applications," Mathematical Inequalities \& Applications, vol. 9, no. 1, pp. 103-106, 2006.

[9] Y. Nishizawa, "Sharpening of Jordan's type and Shafer-Fink's type inequalities with exponential approximations," Applied Mathematics and Computation, vol. 269, pp. 146-154, 2015.

[10] L. Zhang and X. Ma, "New refinements and improvements of Jordan's inequality," Mathematics, vol. 6, no. 12, pp. 1-8, 2018.

[11] S.-P. Zeng and Y.-S. Wu, "Some new inequalities of Jordan type for sine," The Scientific World Journal, vol. 2013, Article ID 834159, 5 pages, 2013.

[12] F. Qi, "Extensions and sharping s of Jordan's and Kober's inequality," Journal of Mathematics for Technology, vol. 12, no. 4, pp. 98-102, 1996.
[13] B. Maleśević, T. Lutovac, M. Raśajski, and C. Mortici, "Extensions of the natural approach to refinements and generalizations of some trigonometric inequalities," Advances in Difference Equations, vol. 2018, no. 1, p. 90, 2018.

[14] B. A. Bhayo and J. Sándor, “On Jordan's and Kober's inequality," Acta Et Commentationes Universitatis Tartuensis De Mathematica, vol. 20, no. 2, pp. 111-116, 2016.

[15] L. Zhang and X. Ma, "New polynomial bounds for Jordan's and Kober's inequalities based on the interpolation and approximation method," Mathematics, vol. 7, no. 8, pp. 1-9, 2019.

[16] X. Zhang, G. Wang, and Y. Chu, "Extensions and sharpenings of Jordan's and Kober's inequalities," Journal of Inequalities in Pure and Applied Mathematics, vol. 7, no. 7, 2006.

[17] D. S. Mitrinovic, Analytic Inequalties, Springer, New York, NY, USA, 1970.

[18] M. Nenezic and L. Zhu, "Some improvements of JordanSteckin and Becker-Stark inequalities," Applicable Analysis and Discrete Mathematics, vol. 12, no. 1, 2018.

[19] G. Bercu, "The natural approach of trigonometric inequalities-padé approximant," Journal of Mathematical Inequalities, vol. 11, no. 1, pp. 181-191, 2017.

[20] Z. Zhang, H. Shan, and L. Chen, "Refining trigonometric inequalities by using padé approximant," Journal of Inequalities \& Applications, vol. 2018, no. 1, p. 149, 2018.

[21] G. A. Baker and P. Graves-Morris, Padé Approximant, Cambridge University Press, New York, NY, USA, 1996.

[22] C. Berzinski and M. Redivo-Zaglia, "New representations of padé, padé-type, and padé approximants," Journal of Computational and Applied Mathematics, vol. 284, pp. 69-77, 2015.

[23] G. Bercu, "Padé approximant related to remarkable inequalities involving trigonometric functions," Journal of Mathematical Inequalities, vol. 2016, no. 1, p. 99, 2016.

[24] G. Bercu and S. Wu, "Refinements of certain hyperbolic inequalities via the Padé approximation method," Journal of Nonlinear Sciences and Applications, vol. 9, no. 6, pp. 50115020, 2016.

[25] P. J. Davis, Interpolation and Approximation, Dover Publications, New York, NY, USA, 1975. 